\title{
Reversible electrochemical monitoring of surface confined reactions at liquid-liquid interfaces by modulation of ion transfer fluxes
}

\author{
Yida Xu, ${ }^{a}$ Roland De Marco, ${ }^{b}$ Alexey Shvarev ${ }^{a}$ and Eric Bakker*a \\ Received (in Cambridge, UK) 10th March 2005, Accepted 20th April 2005 \\ First published as an Advance Article on the web 11th May 2005 \\ DOI: 10.1039/b503548a
}

The adsorption of the neutral surfactant Brij35 at a liquidliquid interface is reversibly monitored via its disturbance of an electrochemically imposed ion flux across the interface, forming a promising experimental tool for the detection of surface confined reactions at such liquids and polymers.

The zero current potentiometric response of ion-selective electrodes is traditionally thought to be independent of molecular adsorption processes at the membrane surface. The Nernst equation is formulated on the basis of the ion activities in the respective phase boundaries, and an increase in the charge transfer resistance should not influence these values. Instead, electrochemical impedance spectroscopy (EIS) was used to study charge transfer resistance changes at ion-selective electrode membranes, first by Buck $^{1}$ and later by others, including Horvai et al. ${ }^{2}$ and Mikhelson et al. ${ }^{3}$ Successful systems included membranes with additives such as non-ionic surfactants, ${ }^{4}$ membranes with low concentrations of ion-exchangers, ${ }^{5}$ or samples containing discriminated ions. ${ }^{3}$

Here, we introduce and evaluate a direct and reversible sensing principle for the detection of surface adsorption processes at liquid-liquid interfaces. This may develop into an attractive labelfree screening technique that mimics the chemical behavior at cell surfaces. An ion flux of defined magnitude and duration is galvanostatically imposed across a polymeric ion-selective membrane. As Fig. 1 shows, the resulting flux-induced membrane potential may be perturbed as a result of a surface binding event, and may thereby become detectable.

Janata et al. recently used cyclic voltammetry on anion-sensitive conducting polymers and observed that the voltammograms were perturbed upon sample DNA hybridizing with complementary target DNA covalently attached to the surface. ${ }^{6}$ This approach is analogous to that used with metal electrodes, where the electrochemistry of a well defined redox marker can be perturbed by surface binding events.

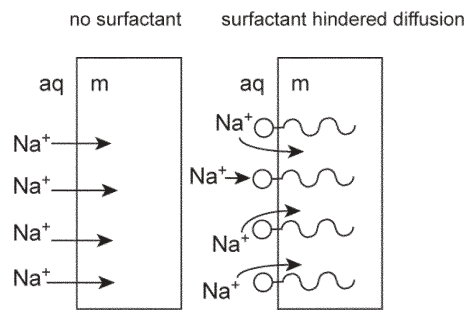

Fig. 1 Scheme showing how the adsorption process at a liquid-liquid interface may alter the galvanostatically induced diffusion/partitioning kinetics of ions for which the membrane is highly selective.

Very recently, Muslinkina and Pretsch explored calciumselective membranes doped with the non-ionic surfactants sorbitan monostearate and sorbitan monopalmitate with zero current potentiometry and EIS. ${ }^{4}$ The membrane electrodes were formulated to induce a continuous inward flux of calcium ions from the sample to the membrane phase, giving a so-called super-Nernstian potential change at a critical calcium activity. Membranes doped with non-ionic surfactants showed charge transfer resistance changes as observed by EIS. Interestingly, however, they also gave a shift of the super-Nernstian potential region when measured potentiometrically, indicating a change in the calcium uptake kinetics of the membrane. These results are quite promising in view of the development of a sensing principle for such surface adsorption processes, but there are drawbacks. The surfactant was observed to significantly reduce the ion selectivity of the membrane in agreement with earlier work, ${ }^{7}$ with higher concentrations generally giving a reduced super-Nernstian response jump. Therefore, the critical activity at which this jump occurs may have been co-influenced by the selectivity change, making interpretation difficult. Moreover, this super-Nernstian response region is, in zero current potentiometry, difficult to reproduce. The transient ion extraction region of interest is strongly influenced by the diffusion layer thicknesses and compositions in both phases, which change continuously and uncontrollably. Indeed, the authors appear to have preferred the use of EIS to study such surfactant adsorption effects, and, in subsequent work, the recognition of concanavalin A at a surfactant covered ion-selective membrane. ${ }^{8}$

Here, we apply pulsed galvanostatic chronopotentiometry, a non-equilibrium method, to directly monitor the surface blocking effect induced by the hydrophilic non-ionic surfactant Brij35 at the surface of a sodium-selective polymeric membrane. $\uparrow$ As recently established, this technique gives potential readings that are very similar to those observed in zero current potentiometry, but affords accurate electrochemical control of the ion extraction process. ${ }^{9}$ This method also eliminates memory effects relative to cyclic voltammetry because any ions extracted into the membrane during a particular measurement are again quantitatively expelled. ${ }^{10}$ Consequently, the super-Nernstian response region mentioned above is normally stable and fully reproducible. ${ }^{9}$ Here, electrochemical impedance spectroscopy is used as an established control technique to corroborate the data.

The non-ionic surfactant Brij35 was chosen here because it may be conveniently added to the sample solution. The sensor response before and after addition of surfactant may therefore be more conveniently characterized compared to doping the surfactant into the membrane during preparation. ${ }^{4}$ The surfactant Brij35, as with most surfactants of this class, has a tendency not only to adsorb onto the membrane surface but to extract into the bulk of the 
membrane as well. Earlier work by Malinowska and Meyerhoff ${ }^{7}$ revealed that such surfactants may, once extracted into the membrane, act as metal binding chelators for interfering ions, sometimes resulting in diminished ion selectivity of the membrane. Malinowska and Meyerhoff studied Brij35 and found its effect to be smaller than other surfactants, especially with calix[4]arene type receptors used in the membrane. ${ }^{7}$

The sodium response was evaluated in a region where sample depletion occurs at a critical sodium activity, generating a so-called super-Nernstian response region in analogy to recent work reported by Muslinkina and Pretsch. ${ }^{4}$ For this purpose, the sample background electrolyte contained the highly discriminated magnesium ion. Fig. 2A shows the observed potential readings upon successive addition of $\mathrm{NaCl}$ to a background of $0.01 \mathrm{M} \mathrm{MgSO}_{4}$. We used here $1 \mathrm{~s}$ cathodic current pulses of $2 \mu \mathrm{A}$ (current density of $0.25 \mu \mathrm{A} \mathrm{mm}{ }^{-2}$ ), each followed by a 15 s resting pulse in potentiostatic mode at $0 \mathrm{~V} .^{11}$ The potentials, sampled at the end of each current pulse, show the desired super-Nernstian response jump around $\log a_{\mathrm{Na}}=-4.3$, indicating that the background ion magnesium is here sufficiently discriminated.

Subsequently, the effect of Brij35 on the sensor response was explored by repeating the same experiment in samples containing a known concentration of this surfactant ranging from $10^{-6}$ to $10^{-4}$ M. As Fig. 2A shows, the observed potentials in the samples with the lowest sodium concentrations, containing mainly
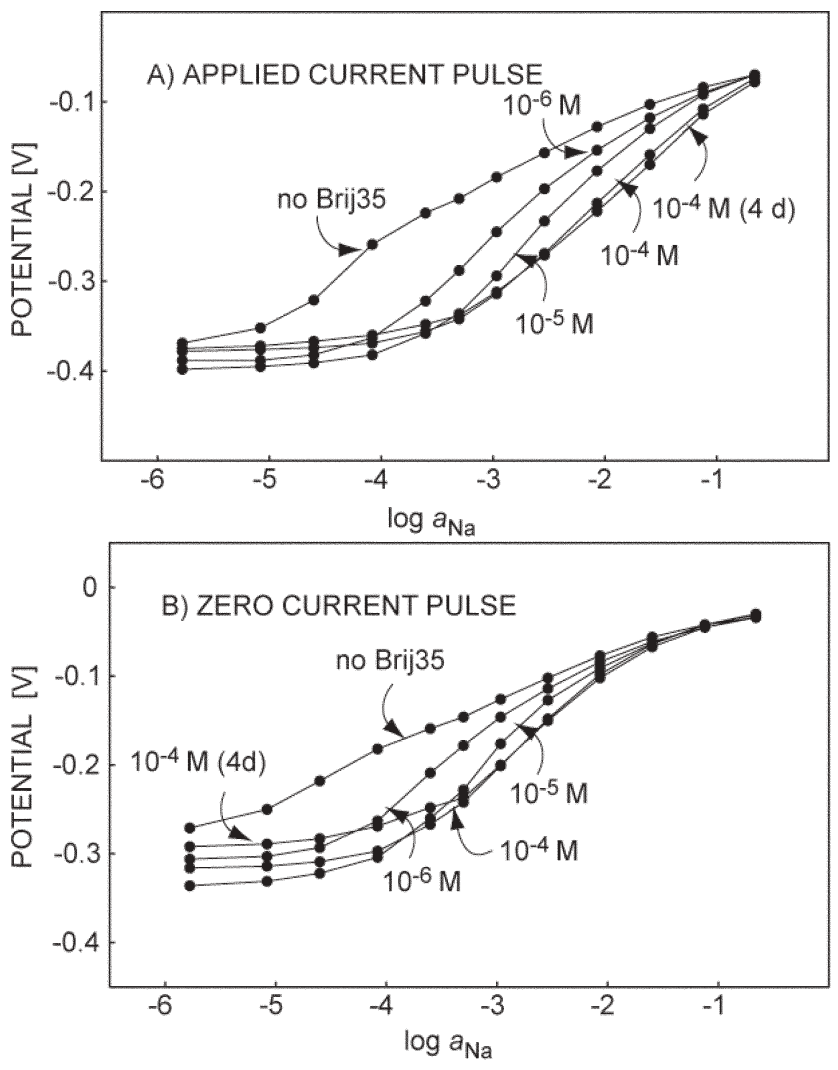

Fig. 2 Pulsed galvanostatic response of a sodium-selective membrane, sampled at the end of (A) a $1 \mathrm{~s}$ cathodic current pulse and (B) a $0.5 \mathrm{~s}$ zero current pulse imposed immediately after the cathodic current pulse. The sample solutions contained $10 \mathrm{mM} \mathrm{MgSO}_{4}$ and the indicated molar concentrations of the surfactant Brij35, including a repeat measurement at the highest concentration after 4 days reconditioning time in $0.1 \mathrm{M} \mathrm{NaCl}$. magnesium sulfate and surfactant, change overall by about $25 \mathrm{mV}$ with increasing surfactant levels. This suggests that the selectivity of the membrane does not appreciably deteriorate in the presence of the surfactant. Also note that the potentials at the highest concentration of sodium in the sample are essentially independent of surfactant concentration, indicating that the ion extraction thermodynamics are not influenced significantly by the surfactant. On the other hand, the so-called super-Nernstian step for the sodium calibration curve shifts to higher concentrations with increasing concentration of Brij35. Since the location of the super-Nernstian step is known to primarily depend on kinetic parameters (diffusion coefficients in both phases and diffusion layer thicknesses), this observed shift can be best explained by a hindered diffusion of sodium ions to or across the membrane interface. The potential-time trace for this experiment is very stable, even in the presence of surfactant (data not shown), which stands in contrast to zero current potentiometry, where the superNernstian region is known to give rise to strong potential instabilities. This is a key advantage afforded by using pulsed galvanostatically controlled sensors, as established previously. ${ }^{9}$ Note that the response experiment shown in Fig. 2A could be quantitatively repeated with the same membrane after conditioning in $0.1 \mathrm{M} \mathrm{NaCl}$ for 4 days (see Fig. 2A). Immediate reproducibility was not as good, owing to the difficulty of rapid removal of the surfactant from the membrane surface after prolonged exposure.

The alternate surfactant Triton X-100 was also evaluated on the same type of membranes and shown to behave similarly, as the apparent super-Nernstian step in the sodium calibration curve was found to shift by about 1 order of magnitude to higher activities in contact with $50 \mu \mathrm{M}$ surfactant. However, this surfactant exhibited a deteriorating effect on the membrane selectivity as evidenced by a $47 \mathrm{mV}$ potential increase in the magnesium background solution, and was therefore not further considered here.

Direct detection of surfactant adsorption is demonstrated in Fig. 3, where Brij35 was added incrementally to a background solution containing $0.01 \mathrm{M} \mathrm{MgSO}_{4}$ and $6 \mathrm{mM} \mathrm{NaCl}$. With increasing Brij35 concentration, the potential first drops gradually, then stabilizes at $c a .1 \times 10^{-4} \mathrm{M}$ Brij35. Since the critical micelle concentration of this surfactant is $9 \times 10^{-5} \mathrm{M},{ }^{12}$ the sample activity of Brij35 above this concentration is no longer expected to

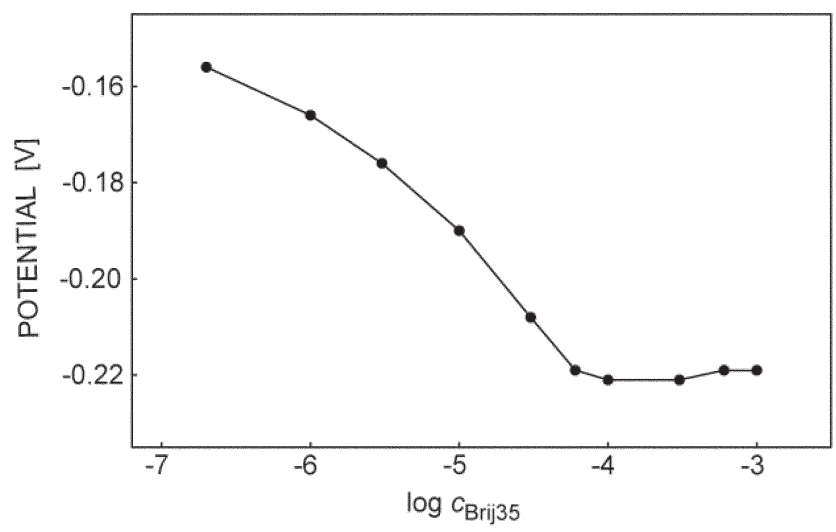

Fig. 3 Pulsed galvanostatic response of a sodium-selective membrane to varying concentrations of Brij35 in $10 \mathrm{mM} \mathrm{MgSO}_{4}$ and $6 \mathrm{mM} \mathrm{NaCl}$, demonstrating direct surfactant detection. The potentials were sampled at the end of a $1 \mathrm{~s}, 2 \mu \mathrm{A}\left(0.25 \mu \mathrm{A} \mathrm{mm}{ }^{-2}\right)$ cathodic current pulse. 
A

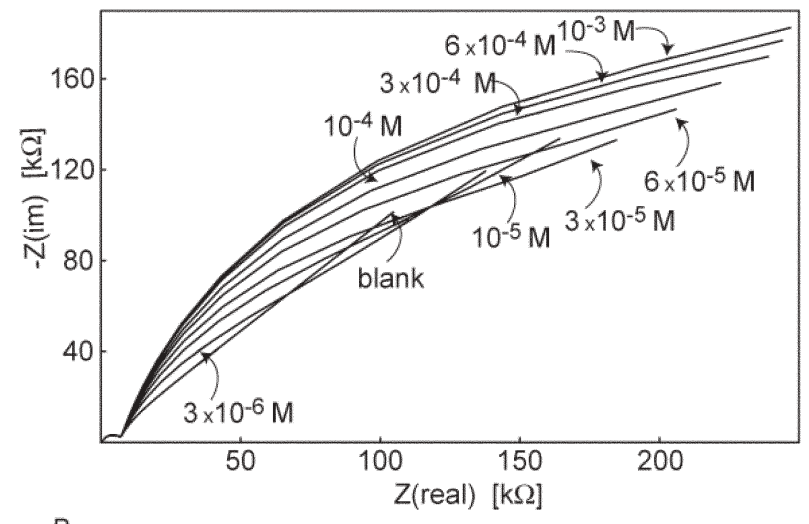

B

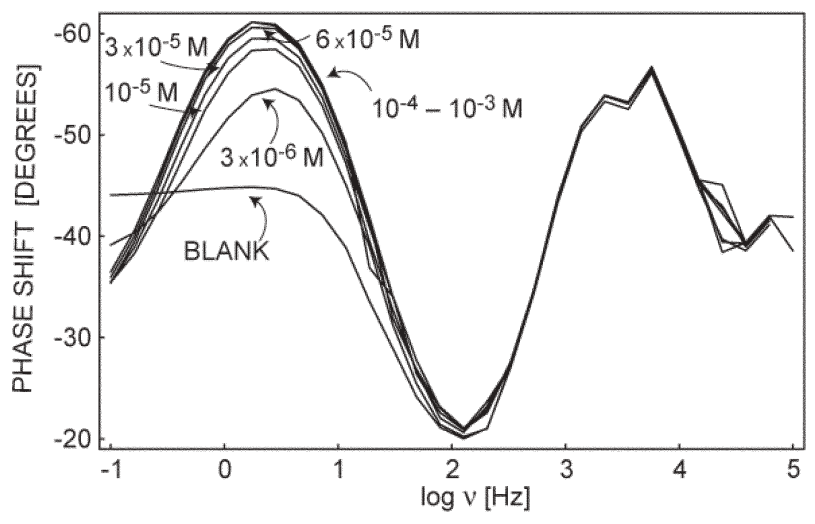

Fig. 4 Electrochemical impedance spectra, represented as (A) Nyquist plots and (B) Bode phase plots, for a sodium-selective membrane in contact with $10 \mathrm{mM} \mathrm{MgSO}_{4}$ and $6 \mathrm{mM} \mathrm{NaCl}$ and different concentrations of Brij35 in the aqueous solution.

increase. This should lead to maximum surface coverage and hence to a maximum potential response.

Comparative EIS experiments were performed to better interpret the pulsed galvanostatic data.t In the respective Nyquist plot (Fig. 4A) for the experiment shown in Fig. 3, the socalled Warburg diffusion impedance is overshadowing the increase in the low frequency charge transfer resistance as a function of surfactant concentration. This gives rise to the apparent trend toward distorted low frequency semicircles which may comprise a convolution of the Warburg diffusion impedance and the ion transfer resistance. ${ }^{13}$ In the corresponding Bode plot (Fig. 4B), the phase shift of the second time constant in the low frequency region is significantly increased upon increasing the Brij35 concentration, which is indicative of surfactant adsorption at the membrane surface. Since the EIS data do not provide unambiguous evidence for an increase in the ion transfer resistance as the prevailing mechanism due to the confounding influence of the Warburg diffusion impedance at low frequency, the results can currently be chiefly explained by changes in ion diffusion resistance induced by surfactant adsorption at the sensor-solution interface. Credence for the influence of surfactant adsorption on the ion flux is provided by the excellent fit of the double layer capacitance data, as obtained from extrapolation of the $\log |Z| v s . \log (2 \pi v)$ impedance plots at $\log (2 \pi v)=0$, to a linearized Langmuir adsorption isotherm plot of $\theta /(1-\theta)$ vs. $c\left(y=94367 x ; r^{2}=0.9978\right)$. The adsorption isotherm of Brij35 was recently studied independently by surface tension measurements at the water-hexane interface. ${ }^{14}$ The surface tension continuously decreased from $10^{-6} \mathrm{M}$ to $10^{-4} \mathrm{M}$ Brij35, after which it leveled off, in analogy to the data shown in Fig. 3.

Additional evidence of this diffusion controlled mechanism comes from the recorded potentials for a $0.5 \mathrm{~s}$ zero current pulse applied immediately after each applied current pulse in the same experiment as in Fig. 2A and shown in Fig. 2B. This measurement protocol was recently introduced ${ }^{11}$ and is useful here because $I R$ drops across the cell are eliminated during this pulse. If a charge transfer resistance increase upon surfactant adsorption would be the prevailing mechanism, the observed potentials during this pulse would not be expected to change appreciably. Fig. 2B, however, again shows a super-Nernstian response step that shifts to higher sodium concentrations with increasing surfactant levels. This can again be best explained with an increase in the sodium diffusion hindrance caused by the adsorption of surfactant onto the membrane surface. It is anticipated that such pulsed galvanostatic experiments will form a promising protocol to probe surface binding reactions at liquid-liquid interfaces.

We acknowledge the National Institutes of Health (GM071623 and EB002189) and the Australian Research Council (ARC) for financial support of this research.

Yida Xu, ${ }^{a}$ Roland De Marco, ${ }^{b}$ Alexey Shvarev ${ }^{a}$ and Eric Bakker*a ${ }^{a}$ Department of Chemistry and Biochemistry, Auburn University, Auburn,.AL36849,USA.E-mail: eric.bakker@auburn.edu ${ }^{b}$ Department of Applied Chemistry, Curtin University, Perth, WA 6845, Australia.E-mail:R.DeMarco@curtin.edu.au

\section{Notes and references}

$\uparrow$ The sodium-selective membrane was solvent cast as described ${ }^{9}$ and contained $10 \mathrm{mmol} \mathrm{kg}$ sodium ionophore tert-butyl calix[4]arene tetraacetic acid tetraethylester, $10 \mathrm{wt} \%$ inert lipophilic electrolyte tetradodecylammonium tetrakis(4-chlorophenyl)borate (ETH 500), and the plasticizer $o$-nitrophenyloctylether and the polymer poly(vinyl chloride) in a 2 : 1 mass ratio. An $8 \mathrm{~mm}^{2}$ membrane disk was cut from this parent membrane, mounted in a Philips electrode body, backfilled with $0.1 \mathrm{M} \mathrm{NaCl}$ and conditioned in $0.1 \mathrm{M} \mathrm{NaCl}$ overnight before performing the electrochemical experiments in a described three electrode cell. ${ }^{9}$

$\$$ All EIS studies were undertaken using a Princeton Applied Research PARSTAT 2263 instrument. Experimental control and data acquisition was performed using a personal computer running the PowerSINE software. EIS spectra were collected at the open circuit potential using an AC amplitude of $\pm 10 \mathrm{mV} \mathrm{rms}$ and a frequency range of $100 \mathrm{kHz}-100 \mathrm{mHz}$.

1 R. P. Buck, Ion-Sel. Electrode Rev., 1982, 4, 3.

2 G. Horvai, E. Gráf, K. Tóth, E. Pungor and R. P. Buck, Anal. Chem., 1986, 58, 2735.

3 K. N. Mikhelson, J. Bobacka, A. Lewenstam and A. Ivaska, Electroanalysis, 2001, 13, 876.

4 L. Muslinkina and E. Pretsch, Electroanalysis, 2004, 16, 1569.

5 R. D. Armstrong, J. C. Lockhart and M. Todd, Electrochim. Acta, 1986, 31, 591.

6 L. A. Thompson, J. Kowalik, M. Josowicz and J. Janata, J. Am. Chem. Soc., 2003, 125, 324.

7 E. Malinowska and M. E. Meyerhoff, Anal. Chem., 1998, 70, 1477.

8 L. Muslinkina and E. Pretsch, Chem. Commun., 2004, 10, 1218.

9 A. Shvarev and E. Bakker, Anal. Chem., 2003, 75, 4541.

10 S. Jadhav, A. J. Meir and E. Bakker, Electroanalysis, 2000, 12, 1251.

11 S. Makarychev-Mikhailov, A. Shvarev and E. Bakker, J. Am. Chem. Soc., 2004, 126, 10548.

12 A. Wangsakan, P. Chinachoti and D. J. McClements, Langmuir, 2004, 20, 3913.

13 K. Tóth, E. Gráf, G. Horvai, E. Pungor and R. P. Buck, Anal. Chem., 1986, 58, 274.

14 L. Liggieri, M. Ferrari, D. Mondelli and F. Ravera, Faraday Discuss., 2005, 129, 125. 